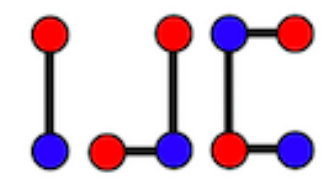

\title{
Graceful labeling on torch graph
}

\author{
Jona Martinus Manulang, Kiki Ariyanti Sugeng \\ Universitas Indonesia, Kampus UI Depok, 16424, Indonesia \\ jona.martinus@sci.ui.ac.id,kiki@sci.ui.ac.id
}

\begin{abstract}
Let $G$ be a graph with vertex set $V=V(G)$ and edge set $E=E(G)$. An injective function $f: V \rightarrow\{0,1,2, \ldots,|E|\}$ is called graceful labeling if $f$ induces a function $f^{*}(u v)=|f(u)-f(v)|$ which is a bijection from $E(G)$ to the set $\{1,2,3, \ldots,|E|\}$. A graph which admits a graceful labeling is called a graceful graph. In this paper, we show that torch graph $O_{n}$ is a graceful graph.

Keywords: graceful graph, graceful labeling, torch graph

Mathematics Subject Classification: 05C78

DOI: $10.19184 /$ ijc.2018.2.1.2
\end{abstract}

\section{Introduction}

In this paper we consider only finite simple undirected graphs. The set of vertices and edges of a graph $G$ will be denoted by $V(G)$ and $E(G)$, respectively. A labeling of a graph $\mathrm{G}$ is a function that carries a set of graph elements, usually the vertices and/or edges, into a set of numbers, usually integers, called labels. A graceful labeling of a graph $G=(V, E)$ is an injective function $f$ from the vertices of $G$ to the set $\{0,1,2, \ldots,|E|\}$ such that when each edge $u v$ is assigned the label $|f(u)-f(v)|$, the resulting edge label are distinct. Rosa [3] has identified essentially three reasons why a graph fails to be graceful: (1) $G$ has too many vertices and not enough edges, (2) $G$ has too many edges, and (3) $G$ has the wrong parity. A graph which admits a graceful labeling is called a graceful graph. Many results of graceful labelings can be found in [2].

In [1] Rachmandika and Sugeng proved that Torch graph $O_{n}$ has super edge-magic total labeling. The aim of this paper is to prove that torch graph $O_{n}$ is graceful graph. In the next section we give the definition of Torch graph $O_{n}$. We give main result in Section 3.

Received: 05 Oct 2017, Revised: 12 Feb 2018, Accepted: 11 Apr 2018. 


\section{Torch Graph}

Torch Graph $O_{n}$ has $n+4$ vertices and $2 n+3$ edges. The set of vertices and edges, respectively, are :

$V\left(O_{n}\right)=\left\{v_{i} \mid 1 \leq i \leq n+4\right\}$

$E\left(O_{n}\right)=\left\{v_{i} v_{n+1} \mid 2 \leq i \leq n-2\right\} \cup\left\{v_{i} v_{n+3} \mid 2 \leq i \leq n-2\right\} \cup\left\{v_{1} v_{i} \mid n \leq i \leq n+4\right\} \cup$ $\left\{v_{n-1} v_{n}, v_{n} v_{n+2}, v_{n} v_{n+4}, v_{n+1} v_{n+3}\right\}$

The following Figure 1 shows a Torch Graph $\mathrm{O}_{3}$.

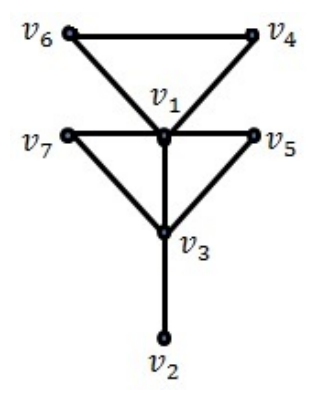

Figure 1. Torch Graph $O_{3}$

The following Figure 2 shows a Torch Graph $O_{n}$ for $n \geq 4$.

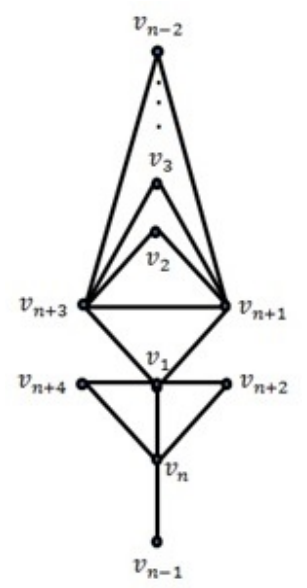

Figure 2. Torch Graph $O_{n}$ for $n \geq 4$

\section{Main Result}

In this section we give a proof that Torch graph $O_{n}$ is a graceful Graph for $n \geq 3$. 
Theorem 3.1. Torch graph $O_{n}$ is a graceful graph for $n \geq 3$.

Proof. The number of vertices in a torch graph $O_{n}$ is $n+4$ and the number of edges is $2 n+3$. We will show that there exists injective function $f$ from $V\left(O_{n}\right)$ to $\{0,1,2, \ldots, 2 n+3\}$ such that $f$ induces bijective function $f^{*}$ from $E\left(O_{n}\right)$ to $\{1,2, \ldots, 2 n+3\}$.

Let $f$ be the function on $V\left(O_{n}\right)$ defined by :

$$
f\left(v_{i}\right)= \begin{cases}0 & \text { for } i \in\{1\}, \\ 2 i-1 & \text { for } i \in\{2,3,4, \ldots, n+2\}, \\ 2(i-3) & \text { for } i \in\{n+3, n+4\} .\end{cases}
$$

Since all values of $f\left(v_{i}\right)$ are distinct and $0 \leq f\left(v_{i}\right) \leq 2 n+3$, we conclude that $f$ is an injective function from $V\left(O_{n}\right)$ to $\{0,1,2, \ldots, 2 n+3\}$.

Now we will proof that $f^{*}$ is bijective. Note that :

- For $v_{i} v_{j} \in\left\{v_{i} v_{j} \mid i=2,3, \ldots, n-2\right.$ and $\left.j=n+3\right\}$ we have :

$$
\begin{aligned}
f^{*}\left(v_{i} v_{j}\right) & =\left|f\left(v_{i}\right)-f\left(v_{j}\right)\right| \\
& =|(2 i-1)-2(n+3-3)| \\
& =|2 i-2 n-1| \\
& =2 n-2 i+1 .
\end{aligned}
$$

- For $v_{i} v_{j} \in\left\{v_{i} v_{j} \mid i=1\right.$ and $\left.j=n, n+1, n+2\right\}$ we have :

$$
\begin{aligned}
f^{*}\left(v_{i} v_{j}\right) & =\left|f\left(v_{i}\right)-f\left(v_{j}\right)\right| \\
& =|0-(2 j-1)| \\
& =|-2 j+1| \\
& =2 j-1 .
\end{aligned}
$$

- For $v_{i} v_{j} \in\left\{v_{i} v_{j} \mid i=1\right.$ and $\left.j=n+3, n+4\right\}$ we have :

$$
\begin{aligned}
f^{*}\left(v_{i} v_{j}\right) & =\left|f\left(v_{i}\right)-f\left(v_{j}\right)\right| \\
& =|0-2(j-3)| \\
& =|-2 j+6| \\
& =2 j-6 .
\end{aligned}
$$

- For another $v_{i} v_{j}$ we have :

$$
\begin{aligned}
f^{*}\left(v_{n-1} v_{n}\right) & =\left|f\left(v_{n-1}\right)-f\left(v_{n}\right)\right| \\
& =|2(n-1)-1-(2 n-1)| \\
& =|2 n-2-1-2 n+1| \\
& =2 .
\end{aligned}
$$




$$
\begin{aligned}
f^{*}\left(v_{n} v_{n+2}\right) & =\left|f\left(v_{n}\right)-f\left(v_{n+2}\right)\right| \\
& =|2 n-1-(2(n+2)-1)| \\
& =|2 n-1-2 n-3| \\
& =4 . \\
f^{*}\left(v_{n} v_{n+4}\right) & =\left|f\left(v_{n}\right)-f\left(v_{n+4}\right)\right| \\
& =|2 n-1-(2(n+4-3))| \\
& =|2 n-1-2 n-8+6| \\
& =3 . \\
& =\left|f\left(v_{n+1}\right)-f\left(v_{n+3}\right)\right| \\
& =|2(n+1)-1-(2(n+3-3))| \\
& =|2 n+2-1-2 n| \\
& =1 .
\end{aligned}
$$

Thus we obtain :

$$
f^{*}\left(v_{i} v_{j}\right)= \begin{cases}2 n-2 i+2 & \text { for }\left\{v_{i} v_{j} \mid i=2,3, \ldots, n-2 \text { and } j=n+1\right\} \\ 2 n-2 i+1 & \text { for }\left\{v_{i} v_{j} \mid i=2,3, \ldots, n-2 \text { and } j=n+3\right\} \\ 2 j-1 & \text { for }\left\{v_{i} v_{j} \mid i=1 \text { and } j=n, n+1, n+2\right\} \\ 2 j-6 & \text { for }\left\{v_{i} v_{j} \mid i=1 \text { and } j=n+3, n+4\right\} \\ 1 & \text { for } i=n+1 \text { and } j=n+3 \\ 2 & \text { for } i=n-1 \text { and } j=n \\ 3 & \text { for } i=n \text { and } j=n+4 \\ 4 & \text { for } i=n \text { and } j=n+2 .\end{cases}
$$

We can see that :

$f^{*}\left(\left\{v_{i} v_{j} \mid i=2,3, \ldots, n-2\right.\right.$ and $\left.\left.j=n+1\right\}\right)=\{6,8,10, \ldots, 2 n-2\}$.

$f^{*}\left(\left\{v_{i} v_{j} \mid i=2,3, \ldots, n-2\right.\right.$ and $\left.\left.j=n+3\right\}\right)=\{5,7,9, \ldots, 2 n-3\}$.

$f^{*}\left(\left\{v_{i} v_{j} \mid i=1\right.\right.$ and $\left.\left.j=n, n+1, n+2\right\}\right)=\{2 n-1,2 n+1,2 n+3\}$.

$f^{*}\left(\left\{v_{i} v_{j} \mid i=1\right.\right.$ and $\left.\left.j=n+3, n+4\right\}\right)=\{2 n, 2 n+2\}$.

And for another $v_{i} v_{j}$ we have $f^{*}\left(\left\{v_{i} v_{j}\right\}\right)=\{1,2,3,4\}$.

Then we have range of $f^{*}$

$=f^{*}\left(\left\{v_{i} v_{j} \mid i=2,3, \ldots, n-2\right.\right.$ and $\left.\left.j=n+1\right\}\right) \cup f^{*}\left(\left\{v_{i} v_{j} \mid i=2,3, \ldots, n-2\right.\right.$ and $\left.\left.j=n+3\right\}\right) \cup$ $f^{*}\left(\left\{v_{i} v_{j} \mid i=1\right.\right.$ and $\left.\left.j=n, n+1, n+2\right\}\right) \cup f^{*}\left(\left\{v_{i} v_{j} \mid i=1\right.\right.$ and $\left.\left.j=n+3, n+4\right\}\right)$

$=\{6,8,10, \ldots, 2 n-2\} \cup\{5,7,9, \ldots, 2 n-3\} \cup\{2 n-1,2 n+1,2 n+3\} \cup\{2 n, 2 n+2\} \cup\{1,2,3,4\}$

$=\{1,2,3, \ldots, 2 n+3\}$

$=\left\{1,2,3, \ldots,\left|E\left(O_{n}\right)\right|\right\}$. 
This implies that $f^{*}$ is onto and one-one, so we conclude that $f^{*}$ is a bijection from $E\left(O_{n}\right)$ to $\left\{1,2,3, \ldots,\left|E\left(O_{n}\right)\right|\right\}$. Since we have proved that there exist an injective function $f: V\left(O_{n}\right) \rightarrow$ $\left\{0,1,2, \ldots,\left|E\left(O_{n}\right)\right|\right\}$ such that $f$ induces a function $f^{*}(u v)=|f(u)-f(v)|$ which is a bijection from $E\left(O_{n}\right)$ to the set $\left\{1,2,3, \ldots,\left|E\left(O_{n}\right)\right|\right\}$, then we conclude that $O_{n}$ is graceful graph.

The following Figure 3 shows a graceful labeling for $O_{3}$.

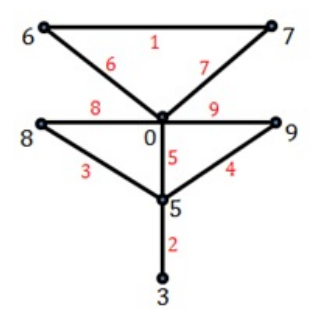

Figure 3. Graceful labeling for $\mathrm{O}_{3}$

The following Figure 4 is an example of graceful labeling for $O_{7}$.

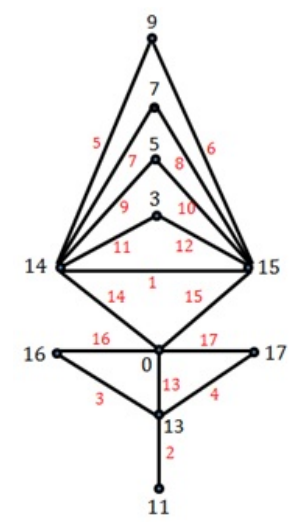

Figure 4. Graceful labeling for $O_{7}$

\section{Acknowledgement}

This research is partially supported by PITTA UI 2017 Research Grant Universitas Indonesia.

\section{References}

[1] P. Rachmandika and K.A.Sugeng, Super edge-magic total labeling of Torch Graph, Prosiding Seminar Nasional Matematika. (2014).

[2] J.A. Gallian, A Dynamic Survey of Graph Labeling, Electronic Journal of Combinatorics 19 DS6 (2016) 


\section{Graceful labeling on torch graph \\ J. M. Manulang and K. A. Sugeng}

[3] A. Rosa, On certain valuations of the vertices of a graph, Theory of Graphs (Internat. Symposium, Rome, July 1966), Gordon and Breach, N. Y. and Dunod Paris (1967) 349-355. 